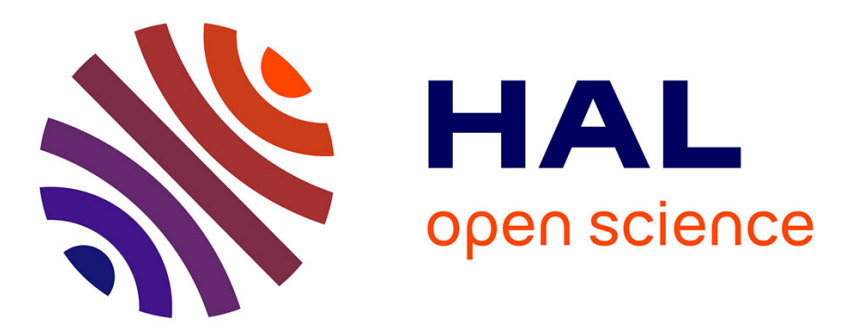

\title{
Strong Coupling in Conserved Surface Roughening: A New Universality Class?
}

Fernando Caballero, Cesare Nardini, Frederic van Wijland, Michael E Cates

\section{To cite this version:}

Fernando Caballero, Cesare Nardini, Frederic van Wijland, Michael E Cates. Strong Coupling in Conserved Surface Roughening: A New Universality Class?. Physical Review Letters, 2018, 121 (2), pp.020601. 10.1103/PhysRevLett.121.020601 . hal-01871379

\section{HAL Id: hal-01871379 https://hal.science/hal-01871379}

Submitted on 10 Sep 2018

HAL is a multi-disciplinary open access archive for the deposit and dissemination of scientific research documents, whether they are published or not. The documents may come from teaching and research institutions in France or abroad, or from public or private research centers.
L'archive ouverte pluridisciplinaire HAL, est destinée au dépôt et à la diffusion de documents scientifiques de niveau recherche, publiés ou non, émanant des établissements d'enseignement et de recherche français ou étrangers, des laboratoires publics ou privés. 


\title{
Strong Coupling in Conserved Surface Roughening: A New Universality Class?
}

\author{
Fernando Caballero, ${ }^{1, *}$ Cesare Nardini, ${ }^{2, \dagger}$ Frédéric van Wijland, ${ }^{3, \sharp}$ and Michael E. Cates ${ }^{1}$ \\ ${ }^{1}$ DAMTP, Centre for Mathematical Sciences, University of Cambridge, Wilberforce Road, \\ Cambridge CB3 OWA, United Kingdom \\ ${ }^{2}$ Service de Physique de l'État Condensé, CNRS UMR 3680, CEA-Saclay, 91191 Gif-sur-Yvette, France \\ ${ }^{3}$ Laboratoire Matière et Systèmes Complexes, UMR 7057 CNRS/P7, Université Paris Diderot, \\ 10 rue Alice Domon et Léonie Duquet, 75205 Paris cedex 13, France
}

(Received 28 March 2018; revised manuscript received 8 June 2018; published 11 July 2018)

\begin{abstract}
The Kardar-Parisi-Zhang (KPZ) equation defines the main universality class for nonlinear growth and roughening of surfaces. But under certain conditions, a conserved KPZ equation (CKPZ) is thought to set the universality class instead. This has non-mean-field behavior only in spatial dimension $d<2$. We point out here that CKPZ is incomplete: It omits a symmetry-allowed nonlinear gradient term of the same order as the one retained. Adding this term, we find a parameter regime where the one-loop renormalization group flow diverges. This suggests a phase transition to a new growth phase, possibly ruled by a strongcoupling fixed point and thus described by a new universality class, for any $d>1$. In this phase, numerical integration of the model in $d=2$ gives clear evidence of non-mean-field behavior.
\end{abstract}

DOI: 10.1103/PhysRevLett.121.020601

Kinetic roughening phenomena arise when an interface is set into motion in the presence of fluctuations. The earliest theoretical investigations [1-3] were concerned with the Eden model [4], originally proposed to describe the shape of cell colonies, and with the ballistic deposition model [5]. Kardar, Parisi, and Zhang (KPZ) [6] discovered an important universality class for growing rough interfaces, by adding the lowest-order nonlinearity to the continuum Edwards-Wilkinson (EW) model, in which height fluctuations are driven by nonconserved noise and relax diffusively [7]. The KPZ equation inspired many analytic, numerical, and experimental studies [8-10] and continues to surprise researchers $[9,11-15]$, not least of all because of a strong-coupling fixed point not accessible perturbatively [6]. Several experiments have been performed [16] to confirm the KPZ universality class and recently gained sufficient statistics to show universal properties beyond scaling laws [17-19]. Finally, how to define solutions of stochastic nonlinear partial differential equations is a well-studied mathematical problem [20,21]; interestingly, recent progress made on the KPZ equation [22] uses a construction related to the renormalization group (RG) [23].

Despite its fame, the KPZ equation does not describe all isotropically roughening surfaces; various other universality classes have been identified $[8,24]$. In particular, it is agreed that, in some cases such as vapor deposition and idealized molecular beam epitaxy [25], surface roughening should be described by conservative dynamics (rearrangements dominate any incoming flux), with no leading-order correlation between the hopping direction and the local slope. These considerations eliminate the EW linear diffusive flux and make the geometric nonlinearity addressed by KPZ not allowed [8]. What remains is a conserved version of the KPZ equation (CKPZ) [26-28], which has been widely studied for nearly three decades $[8,29-32]$ :

$$
\dot{\phi}=-\nabla \cdot \mathbf{J}_{\lambda}+\eta ; \quad \mathbf{J}_{\lambda}=\nabla\left\{\kappa \nabla^{2} \phi+\lambda|\nabla \phi|^{2}\right\}
$$

Here $\phi(\mathbf{r}, t)$ is the height of the surface above point $\mathbf{r}$ in a $d$-dimensional plane, $\mathbf{J}_{\lambda}$ is the deterministic current, and $\eta$ is a Gaussian conservative noise with variance $\left\langle\eta(\mathbf{r}, t) \eta\left(\mathbf{r}^{\prime}, t^{\prime}\right)\right\rangle=-2 D \nabla^{2} \delta^{d}\left(\mathbf{r}-\mathbf{r}^{\prime}\right) \delta\left(t-t^{\prime}\right)$. In the linear limit, $\lambda=0$, Eq. (1) reduces to the Mullins equation for curvature driven growth (a conserved counterpart of EW) [33] whose large-scale behavior is controlled by two exponents, $\chi=(2-d) / 2$ and $z=4$, with spatial and temporal correlators obeying $\left\langle\phi(\mathbf{r}, t) \phi\left(\mathbf{r}^{\prime}, t\right)\right\rangle \sim\left|\mathbf{r}-\mathbf{r}^{\prime}\right|^{2 \chi} \quad$ and $\left\langle\phi(\mathbf{r}, t) \phi\left(\mathbf{r}, t^{\prime}\right)\right\rangle \sim\left|t-t^{\prime}\right|^{2 \chi / z}$. The nonlinear term $\lambda|\nabla \phi|^{2}$ can be interpreted microscopically as a nonequilibrium correction to the chemical potential, causing jump rates to depend on the local steepness at the point of take-off as well as on the curvature [8].

The properties of the CKPZ universality class are well known [8]: The upper critical dimension is 2, above which the RG flow leads to the Gaussian fixed point of the Mullins equation, where $\chi<0$ implies smooth growth. Only for $d<2$ is the nonlinearity relevant; a nontrivial fixed point then emerges perturbatively [see Fig. 3(a)]. The one-loop RG calculation [26,30] shows that, at leading order in $\epsilon=2-d$, the critical exponents are $z=4-\epsilon / 3$ and $\chi=\epsilon / 3$; the surface is now rough $(\chi>0)$. Such predictions turn out to be very accurate when tested against numerical integration of CKPZ [8]. 
In this Letter, we argue that CKPZ is not the most general description of conservative roughening without leadingorder slope bias and that a potentially important universality class may have been overlooked by assuming so. We show this by establishing the importance in $d>1$ of a second, geometrically motivated nonlinear term, also of leading order, whose presence fundamentally changes the structure of the one-loop RG flow, creating a separatrix beyond which the flow runs away to infinity. This might lead to three conclusions: (i) The runaway is an unphysical feature of the one-loop RG flow, cured at higher orders; (ii) the separatrix in the RG flow marks a phase transition towards a new phase, where scale invariance is lost; (iii) scale invariance is present in this new phase, but its properties are dictated by a strong-coupling fixed point. Observe that (iii) closely resembles what is found for KPZ, whose strong-coupling regime is long established. We finally perform numerical simulations in the most physically relevant case of $d=2$ and show evidence that the separatrix is not just an artifact of the one-loop RG flow.

For nonconserved dynamics, the KPZ nonlinearity stands alone at leading order after imposing all applicable symmetries. For conserved dynamics, however, the CKPZ choice $\mathbf{J}_{\lambda}$ of the deterministic current in (1) is not the only one possible. All symmetries consistent with $\mathbf{J}_{\lambda}$ also admit, at the same order $\left(\nabla^{3}, \phi^{2}\right)$, a second term:

$$
\mathbf{J}_{\zeta}=-\zeta\left(\nabla^{2} \phi\right) \nabla \phi
$$

Like any vector field, $\mathbf{J}_{\zeta}$ can be written via Helmholtz decomposition as the sum of a rotational part $\mathbf{J}_{r}$ and an irrotational part $\mathbf{J}_{\text {irr }}$. The rotational part has no effect on $\dot{\phi}$. Although $\mathbf{J}_{\text {irr }}$ can always be written as the gradient of a scalar function, this function need not be a local function of $\phi$ and its gradients, even though $\mathbf{J}_{\zeta}=\mathbf{J}_{r}+\mathbf{J}_{\text {irr }}$ is local in that sense. This expresses the fact that Helmholtz decomposition of a vector field does not commute with its gradient expansion.

The $\zeta$ term can be explained by considering more carefully the "blind jumping" dynamics often used to motivate CKPZ [8]. Specifically, we suppose jumping particles to move a small fixed geodesic distance along the surface in a random direction. To visualize the resulting physics, consider curving a flat sheet of paper into a sinusoidally corrugated surface $a \cos k x$ and then applying a shear deformation in the $(y, \phi)$ plane to give $\phi(x, y)=$ $a \cos k x+b y$. This resembles a sloping roof with alternating ridges and grooves (Fig. 1). The locus of points of constant geodesic distance from some departure point (with $\left.y=y_{0}\right)$ is as shown in Fig. 1(b). We now ask the fraction $f$ of landing sites (i.e., of points on the folded circle) that have $y>y_{0}$. It can be confirmed that $f>1 / 2$ for a point on a ridge $\left(\phi_{x x}<0\right)$, but $f<1 / 2$ for a point in a groove $\left(\phi_{x x}>0\right)$. The resulting bias towards a positive or negative $y$ increment is bilinear in tilt and curvature, vanishing by y

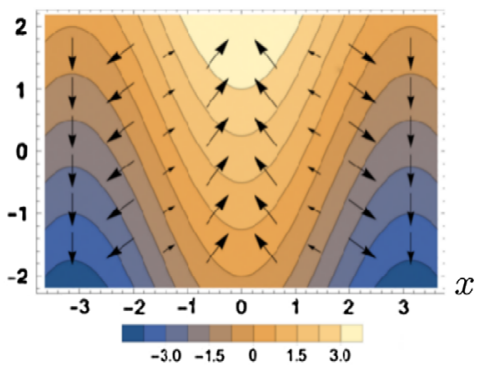

FIG. 1. (a) Contour plot of the surface $\phi(x, y)=2 \cos x+y$ and the current $\mathbf{J}_{\zeta}=-\zeta\left(\nabla^{2} \phi\right) \nabla \phi$ (vectors). $\mathbf{J}_{\zeta}$ resembles a shear flow and thus has nonzero curl. In (b), the blue line is the locus of points $\mathcal{C}$ on the surface equidistant from the origin in the natural metric of the surface. The red dotted line is the intersection between the vertical plane $y=0$ and the surface: Because it does not cut $\mathcal{C}$ in half, it induces $\mathbf{J}_{\zeta} \neq 0$ if a particle in $(0,0)$ jumps with equal probability to any site on $\mathcal{C}$.

symmetry when either $k$ or $b$ is zero. It follows that the local deterministic flux in the $y$ direction contains a term $\sim \phi_{x x} \phi_{y}$ which is not captured by $\lambda$ but demands the existence of the $\zeta$ term. This argument generalizes directly to any case where the "landing rate" depends on the geodesic distance only.

We have thus confirmed that the $\zeta$ term is physical, although of course our "blind geodesic jumping" is not the only possible choice of dynamics. With this choice, the $\zeta$ nonlinearity is purely geometric, arising from the transformation from normal to vertical coordinates. Yet the same is true for the KPZ nonlinearity $[6,8]$.

In summary, for $d>1, \mathrm{CKPZ}$ is an incomplete model. Its generalization, which we call CKPZ+, reads

$$
\dot{\phi}=-\nabla^{2}\left\{\kappa \nabla^{2} \phi+\lambda|\nabla \phi|^{2}\right\}-\nabla \cdot \mathbf{J}_{\zeta}+\eta .
$$

We have seen no previous work on (3) in the literature, except for one study corresponding to a specific combination of the $\lambda$ and $\zeta$ terms [34]. Related models, but containing nonconserved noise and the Edward-Wilkinson diffusive term, have been also considered in Refs. $[35,36]$. A standard dimensional analysis [37] shows both $\lambda$ and $\zeta$ to be perturbatively irrelevant for $d>2$, but this does not preclude important differences in critical behavior between CKPZ and CKPZ + in $d>1$. We now present strong evidence for this outcome, first by analyzing the RG flow perturbatively close to the Gaussian fixed point, where we may hold $\kappa, D$ constant [37], so the RG flow is derived in terms of the reduced couplings $\bar{\lambda}^{2}=\lambda^{2} D / \kappa^{3}$ and $\bar{\zeta}^{2}=\zeta^{2} D / \kappa^{3}$. Transforming (3) into Fourier space with wave vector $\mathbf{q}$ and frequency $\omega$, we have

$$
\phi(\hat{\mathbf{q}})=\phi_{0}(\hat{\mathbf{q}})+\frac{G_{0}(q, \omega)}{2} \int_{\hat{\mathbf{q}}^{\prime}} g\left(\mathbf{q}, \mathbf{q}^{\prime}\right) \phi\left(\hat{\mathbf{q}}^{\prime}\right) \phi\left(\hat{\mathbf{q}}-\hat{\mathbf{q}}^{\prime}\right),
$$


where $\hat{\mathbf{q}}=(\omega, \mathbf{q}), \phi_{0}(\hat{\mathbf{q}})=G_{0}(q, \omega) \eta(\hat{\mathbf{q}}) / q^{2}$, the bare propagator is $G_{0}(q, \omega)=q^{2} /\left(-i \omega+\kappa q^{4}\right)$, and $\eta$ is Gaussian noise with $\left\langle\eta(\hat{\mathbf{q}}) \eta\left(\hat{\mathbf{q}}^{\prime}\right)\right\rangle=2 D q^{2}(2 \pi)^{d+1} \delta^{d+1}\left(\hat{\mathbf{q}}+\hat{\mathbf{q}}^{\prime}\right)$.

In Eq. (4), the nonlinearities $\lambda$ and $\zeta$ enter via a function $g\left(\mathbf{q}, \mathbf{q}^{\prime}\right)$ that, on symmetrizing $\mathbf{q}^{\prime} \leftrightarrow\left(\mathbf{q}-\mathbf{q}^{\prime}\right)$, reads

$$
\begin{aligned}
g\left(\mathbf{q}, \mathbf{q}^{\prime}\right)= & -2 \lambda \mathbf{q}^{\prime} \cdot\left(\mathbf{q}-\mathbf{q}^{\prime}\right) \\
& +\zeta\left(\frac{q^{\prime 2} \mathbf{q} \cdot\left(\mathbf{q}-\mathbf{q}^{\prime}\right)}{q^{2}}+\frac{\left|\mathbf{q}-\mathbf{q}^{\prime}\right|^{2} \mathbf{q} \cdot \mathbf{q}^{\prime}}{q^{2}}\right) .
\end{aligned}
$$

We denote the two-point correlation function of the Mullins equation by $C_{0}\left(\hat{\mathbf{q}}, \hat{\mathbf{q}}^{\prime}\right)=(2 \pi)^{d+1} C_{0}(q, \omega) \delta^{d+1}\left(\hat{\mathbf{q}}+\hat{\mathbf{q}}^{\prime}\right)$, where $C_{0}(q, \omega)=2 D G_{0}(q, \omega) G_{0}(-q,-\omega) / q^{2}$.

It is useful to introduce diagrammatic notation, where a line denotes a zeroth-order field $\phi_{0}$ and the correlation function $C_{0}(q, \omega)$ is represented as a circle between two incoming lines. The vertex reads $\left[G_{0}(q, \omega) / 2\right] \times$ $\int_{\hat{\mathbf{q}}^{\prime}} g\left(\mathbf{q}, \mathbf{q}^{\prime}\right) \phi\left(\hat{\mathbf{q}}^{\prime}\right) \phi\left(\hat{\mathbf{q}}-\hat{\mathbf{q}}^{\prime}\right)$, with $\hat{\mathbf{q}}$ the wave vector entering into the vertex. At one loop, all four diagrams shown in Fig. 2 might contribute, but a number of simplifications occur. First consider the diagram in Fig. 2(d), which could renormalize $D$. Taylor expanding, one finds that the leading contribution is $\mathcal{O}\left(q^{4}\right)$, rendering this irrelevant for small $\epsilon$ and close to the Gaussian fixed point. Next, the two triangular diagrams in Figs. 2(b) and 2(c) might renormalize the couplings $\lambda$ and $\zeta$, but explicit computations [38] show that their contributions exactly cancel out. This can also be shown more directly, by generalizing the argument of Ref. [30]. We note that, while $D$ remains unrenormalized at any order in the perturbation theory, $\lambda$ and $\zeta$ do get renormalized at a higher order. Indeed, this is already known to happen in CKPZ [30].

We conclude that the diagram in Fig. 2(a) is the only nonvanishing one to one loop. Its contributions at the order of $q^{0}$ and $q$ vanish [38], giving a leading-order correction $\mathcal{O}\left(q^{2}\right)$, which renormalizes $\kappa$. Higher terms are irrelevant for small $\epsilon$ and close to the Gaussian fixed point, so we neglect them. The shifted value of $\kappa$ is derived in Ref. [38] as
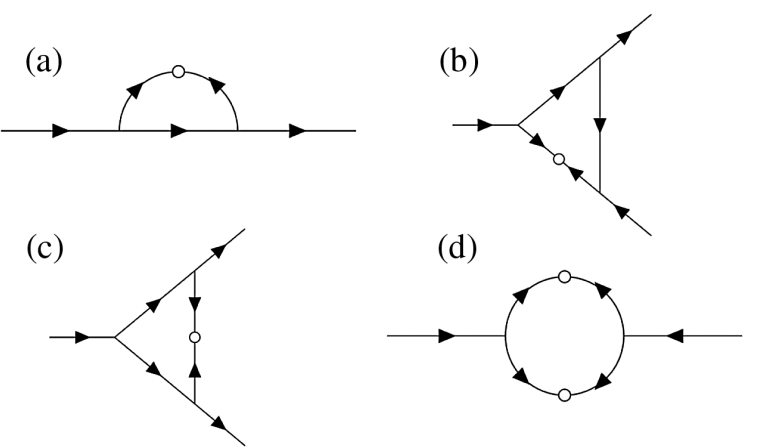

FIG. 2. The diagrams present at one loop. Those in (b)-(d) do not generate any relevant coupling for $\epsilon$ small and close to the Gaussian fixed point.

$$
\kappa_{I}=\kappa\left(1-M(\bar{\lambda}, \bar{\zeta}, d) \frac{S_{d}}{(2 \pi)^{d}} \int_{\Lambda / b}^{\Lambda} x^{d-s 3} d x\right)
$$

where $S_{d}=2 \pi^{d / 2} / \Gamma(d / 2),(\Lambda / b, \Lambda)$ for $b>1$ is the momentum shell integrated out, and

$$
\begin{aligned}
M(\bar{\lambda}, \bar{\zeta}, d)= & \frac{1}{2 d(2+d)}\left[\left(2 d^{2}-3 d-2\right) \bar{\zeta}^{2}\right. \\
& \left.+4 d(d+2) \bar{\lambda} \bar{\zeta}-4(d+2) \bar{\lambda}^{2}\right] .
\end{aligned}
$$

Since the integrating does not produce new relevant couplings at one loop, we are justified in excluding all higher terms from (4) and indeed from the CKPZ+ equation (3).

The last step to obtain the RG flow is rescaling back to the original cutoff $\Lambda$ and reabsorbing all rescalings into the couplings. To do so, one must introduce scaling exponents for time and the field, such that when $q$ is rescaled as $q \rightarrow b q$, then $\omega \rightarrow b^{z} \omega$ and $\phi \rightarrow b^{-\chi} \phi$. The critical exponents $z$ and $\chi$ are fixed by imposing stationarity of the RG flow at its fixed points. This gives the transformation between original and rescaled couplings.

Taking the infinitesimal limit $b=1+d b$, we find the RG flow as

$$
\begin{gathered}
\frac{d \kappa}{d b}=\kappa\left(z-4-M(\bar{\lambda}, \bar{\zeta}, d) \frac{S_{d}}{(2 \pi)^{d}}\right), \\
\frac{d D}{d b}=D(z-2-d-2 \chi), \\
\frac{d(\lambda, \zeta)}{d b}=(\lambda, \zeta)(z+\chi-4) .
\end{gathered}
$$

Consistent with proximity to the Gaussian fixed point, we impose $d \kappa / d b=d D / d b=0$ and use (8)-(10) to obtain

$$
\frac{d(\bar{\lambda}, \bar{\zeta})}{d b}=(\bar{\lambda}, \bar{\zeta})\left(\frac{2-d}{2}+\frac{3}{2} M(\bar{\lambda}, \bar{\zeta}, d) \frac{S_{d}}{(2 \pi)^{d}}\right) .
$$

This, the central technical result of this Letter, is the oneloop RG flow of the CKPZ+ equation (3).

It is now straightforward to obtain the fixed points of the RG flow and their critical exponents setting $d \kappa / d b=$ $d D / d b=0$ and using (8) and (9). First of all, we have the Gaussian fixed point $\lambda=\zeta=0$, whose exponents $z$ and $\chi$ remain those of the Mullins equation mentioned above. In the plane of reduced couplings $(\bar{\lambda}, \bar{\zeta})$, we find additional lines of fixed points on the conics defined by

$$
S_{d} M(\bar{\lambda}, \bar{\zeta}, d)=(2 \pi)^{d}(d-2) / 3 .
$$

Here the critical exponents are those of CKPZ: $z=4-$ $(\epsilon / 3)+\mathcal{O}\left(\epsilon^{2}\right)$ and $\chi=(\epsilon / 3)+\mathcal{O}\left(\epsilon^{2}\right)$. The stability of the $\mathrm{RG}$ fixed points is shown Fig. 3. For $d>2$, the Gaussian 

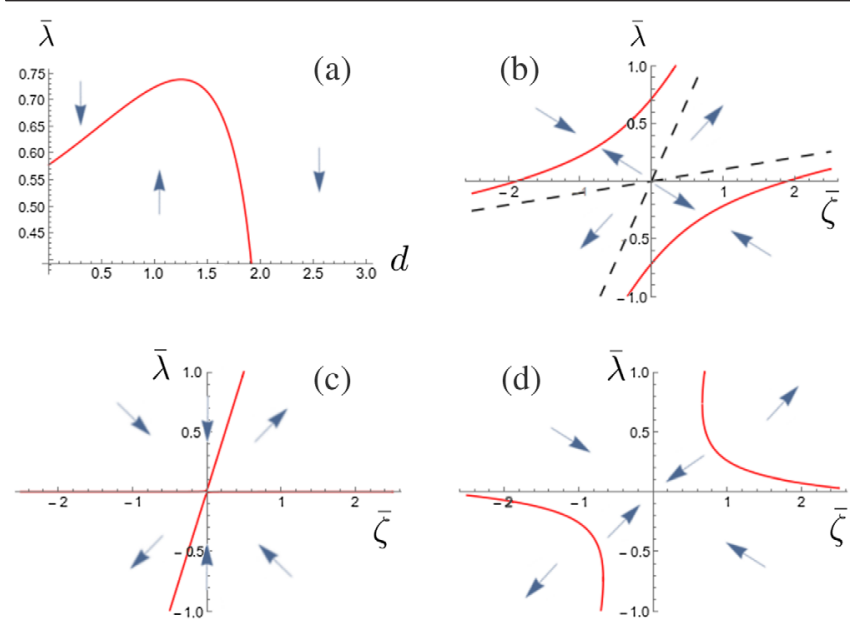

FIG. 3. One-loop RG flow of the (a) CKPZ model as a function of space dimension $d$ and of the CKPZ + model for (b) $1<d<2$, (c) $d=2$, and (d) $d>2$. (CKPZ and CKPZ+ are the same model in $d=1)$. The red lines are the fixed points of the RG flow given by solutions of (12) and the dashed lines their asymptotes; the origin is the Gaussian fixed point. In (b)-(d), the RG flow is radial and its direction is given by the arrows in the plots.

point is locally stable [Fig. 3(d)]. However, the two new lines of fixed points are unstable, and the basin of attraction of the Gaussian fixed point shrinks when approaching $d \rightarrow 2^{+}$. In $d<2$, the Gaussian fixed point becomes unstable, while the lines of fixed points defined by (12) are stable [Figs. 3(a) and 3(b)]. Nonetheless, in $1<d \leq 2$, the latter are not globally attractive, because there are sectors of the reduced couplings plane where the RG flow runs away to infinity. These sectors exclude the pure CKPZ case $(\zeta=0$, vertical axis) so that the runaway is a specific feature of CKPZ + . Similar remarks apply in $d>2$, where the unstable fixed lines are separatrices between the Gaussian fixed point and a runaway to infinity.

The scenario just reported resembles that of KPZ at two loops $[37,39]$. There, the Gaussian fixed point is stable for $d>2$ and unstable for $d<2$. A nontrivial fixed point is again present which is stable for $d<2$ but unstable for $d>2$, where the Gaussian fixed point has a finite basin of attraction, beyond which the flow runs away. In KPZ, this scenario signifies the emergence of a nonperturbative, strong-coupling fixed point $[6,11,39]$, whose existence and properties are by now well established. The two main differences with respect to KPZ are (i) in KPZ, the coupling constant at the non-Gaussian fixed point diverges in the limit $d \rightarrow 2^{-}$[37], and (ii) in KPZ, for $d<2$, the nontrivial fixed point is fully attractive. Despite these differences, it is natural to speculate that the runaway to infinity signifies the presence of a strong-coupling fixed point, with a distinct universality class, also for CKPZ + in $d>1$. However, as anticipated in the introduction, two other scenarios are possible: The runaway to infinity might be just an artifact of the one-loop computation, or the separatrix in the RG flow could signal a phase transition to a different growth phase without scale invariance.

In order to rule out that the runaway of the RG flow is an artifact of the one-loop computation, we performed numerical simulations of (3) in $d=2$, the physically most relevant case. We used a pseudospectral code with a $2 / 3$ dealiasing procedure and a Heun scheme [40] for the time integration. In all simulations, we set $D=\kappa=1$, and all the results shown are obtained starting from a flat initial condition $\phi=0$, but we checked that no difference is obtained when starting from a random initial condition. We checked the stability of our results upon varying the time step in the window $\left(10^{-4}, 5 \times 10^{-3}\right)$. The system sizes used are $L \times L$, with $L$ varying between 15 and 45 . As is standard in the study of roughening surfaces, we report below results on the width of the interface $W(L, t) \equiv\left(1 / L^{2}\right) \int_{\mathbf{r}}\left\langle\phi^{2}(\mathbf{r}, t)\right\rangle$. For fixed $L$, we studied the growth of $W$ with time $t$ and the large-time saturated width.

Within the basin of attraction of the Gaussian fixed point, the code proved numerically stable, allowing us to reproduce the expected critical behavior [38]: $W(t, L) \sim \log t$ and $z=4$. Moreover, simulations in $d=1$ gave exponents agreeing with the known CKPZ values (not shown). In contrast, for parameters where the RG flow diverges to infinity, in order to obtain numerically stable results, we had to add a higher-order regularizer in the form of $k_{6} \nabla^{6} \phi$ in (3). This is irrelevant close to the Gaussian fixed point and does not affect the RG flow there. In Fig. 4, we report $W(L, t)$ as a function of the time for different system sizes. The behavior differs strongly from the mean-field one: After an initial transient, shown in Ref. [38] to depend on $k_{6}, W$ grows much faster than logarithmically. At large enough system sizes, a seemingly size-independent

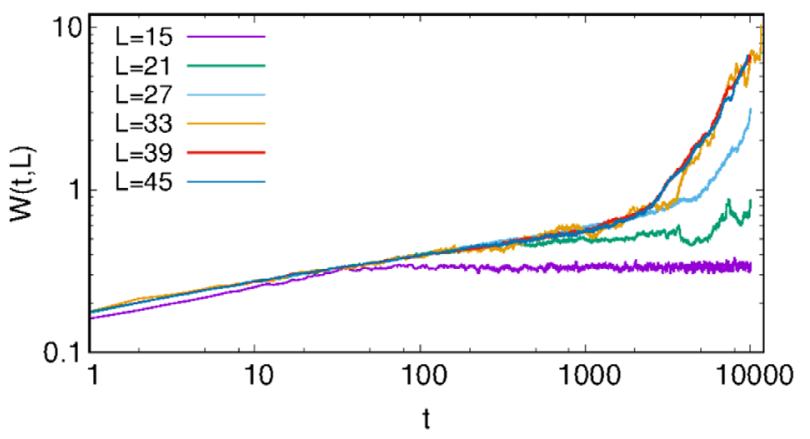

FIG. 4. Growth of the width of the interface $W(L, t)$ with time for different system sizes $L$ of the CKPZ + equation in $d=2$. The parameters $2 \lambda=\zeta=1$ were chosen to lie in the region where the $\mathrm{RG}$ flow diverges. Each line is an average over several noise realizations (from 1600 for $L=15$ to 80 for $L=45$ ). The value of $k_{6}=0.2$. The difference between the present case and the one where the RG flow converges towards the Gaussian fixed point is apparent. Here, at late times (decreasing with $k_{6}$ increasing [38]), the width grows faster than logarithmically. The growth law seems independent from the system size. 
algebraic growth law emerges, although larger $L$ values would be needed to confirm this. Figure 4 is obtained by averaging over many noise realizations (from 1600 for $L=15$ to 400 for $L=45$ ). We report in Ref. [38] the behavior of $W$ for a few individual ones, showing a strong increase in the variance of $W$ with time. This seems to be associated with the late-stage algebraic growth regime.

Our simulations give clear evidence that the runaway to infinity is not an artifact of the one-loop RG flow. For parameters where the RG flow diverges, we observe the formation of localized peaks which then undergo coarsening to give the late-time growth regime reported in Fig. 4 (see Supplemental Movie 1 [38]). This is reminiscent of previous arguments suggesting (albeit for a different growth equation) that the CKPZ + nonlinearities favor mound formation [36]. Moreover, recent analyses of the deterministic version of $\mathrm{CKPZ}+$ in $2 \mathrm{D}$ for a specific combination of $\lambda$ and $\zeta$ concluded that this allows blowup solutions $[35,41]$. It remains an open question whether these solutions persist with noise or are smoothed out, perhaps forming a mound phase or other strong-coupling universality class. It would be interesting to analyze the surface statistics in this regime more quantitatively, as has been done for related growth equations with nonconserved noise [42]. More details on the non-Gaussian growth phase are given in Ref. [38].

In summary, we have argued that the CKPZ equation (1), thought to govern conserved, slope-unbiased roughening dynamics, is incomplete. We introduced a new model, $\mathrm{CKPZ}+(3)$, with a complete set of leading-order nonlinearities. In $d=1$, CKPZ and CKPZ + coincide, but they differ in any $d>1$. Surprisingly, the RG analysis of $\mathrm{CKPZ}+$ at one loop suggests the presence of a non-meanfield growth phase in any dimension $d>1$, which might be due to a new universality class or a loss of scale invariance. Indeed, our numerical analysis clearly indicates that the runaway of the one-loop RG flow signifies new physics at strong coupling.

F. C. is funded by EPSRC DTP IDS studentship, Project No. 1781654. C. N. acknowledges the hospitality provided by DAMTP, University of Cambridge while part of this work was being done. C. N. acknowledges the support of an Aide Investissements d'Avenir du LabEx PALM (ANR10-LABX-0039-PALM). Work was funded in part by the European Resarch Council under the EU's Horizon 2020 Program, Grant No. 760769. M. E. C. is funded by the Royal Society.

*fmc36@cam.ac.uk

†cesare.nardini@gmail.com

frederic.van-wijland@univ-paris-diderot.fr

[1] H. Peters, D. Stauffer, H. Hölters, and K. Loewenich, Z. Phys. B 34, 399 (1979).

[2] M. Plischke and Z. Rácz, Phys. Rev. Lett. 53, 415 (1984).
[3] R. Jullien and R. Botet, J. Phys. A 18, 2279 (1985).

[4] M. Eden, in Symposium on Information Theory in Biology (Pergamon, New York, 1958), pp. 359-370.

[5] F. Family and T. Vicsek, J. Phys. A 18, L75 (1985).

[6] M. Kardar, G. Parisi, and Y.-C. Zhang, Phys. Rev. Lett. 56, 889 (1986).

[7] S. F. Edwards and D. Wilkinson, Proc. R. Soc. A 381, 17 (1982).

[8] J. Krug, Adv. Phys. 46, 139 (1997).

[9] I. Corwin, Random Matrices Theory Appl. 01, 1130001 (2012).

[10] K. A. Takeuchi, Physica A (Amsterdam) 504, 77 (2018).

[11] L. Canet, H. Chaté, B. Delamotte, and N. Wschebor, Phys. Rev. Lett. 104, 150601 (2010).

[12] T. Sasamoto and H. Spohn, Phys. Rev. Lett. 104, 230602 (2010).

[13] T. Kriecherbauer and J. Krug, J. Phys. A 43, 403001 (2010).

[14] B. Derrida, J. Stat. Mech. Theor. Exp. 2007, P07023 (2007).

[15] B. Meerson, E. Katzav, and A. Vilenkin, Phys. Rev. Lett. 116, 070601 (2016).

[16] K. A. Takeuchi, J. Stat. Mech. Theor. Exp. 2014, P01006 (2014).

[17] K. A. Takeuchi, M. Sano, T. Sasamoto, and H. Spohn, Sci. Rep. 1, 34 (2011).

[18] J.-i. Wakita, H. Itoh, T. Matsuyama, and M. Matsushita, J. Phys. Soc. Jpn. 66, 67 (1997).

[19] J. Maunuksela, M. Myllys, O.-P. Kähkönen, J. Timonen, N. Provatas, M. J. Alava, and T. Ala-Nissila, Phys. Rev. Lett. 79, 1515 (1997).

[20] G. Da Prato and J. Zabczyk, Stochastic Equations in Infinite Dimensions (Cambridge University Press, Cambridge, England, 2014).

[21] L. Bertini, N. Cancrini, and G. Jona-Lasinio, Commun. Math. Phys. 165, 211 (1994).

[22] M. Hairer, Ann. Math. 178, 559 (2013).

[23] A. Kupiainen and M. Marcozzi, J. Stat. Phys. 166, 876 (2017).

[24] A.-L. Barabási and H. E. Stanley, Fractal Concepts in Surface Growth (Cambridge University Press, Cambridge, England, 1995).

[25] J. Krim and G. Palasantzas, Int. J. Mod. Phys. B 09, 599 (1995).

[26] T. Sun, H. Guo, and M. Grant, Phys. Rev. A 40, 6763 (1989).

[27] D. Wolf and J. Villain, Europhys. Lett. 13, 389 (1990).

[28] S. Das Sarma and P. Tamborenea, Phys. Rev. Lett. 66, 325 (1991).

[29] M. Constantin, C. Dasgupta, P. Punyindu Chatraphorn, S. N. Majumdar, and S. Das Sarma, Phys. Rev. E 69, 061608 (2004).

[30] H. K. Janssen, Phys. Rev. Lett. 78, 1082 (1997).

[31] Z. Rácz, M. Siegert, D. Liu, and M. Plischke, Phys. Rev. A 43, 5275 (1991).

[32] S. H. Yook, C. K. Lee, and Y. Kim, Phys. Rev. E 58, 5150 (1998).

[33] W. W. Mullins, in Structure, Energetics and Kinetics, edited by N. A. Gjostein and W. D. Robertson (American Society of Metals, Metals Park, OH, 1963).

[34] C. Escudero and E. Korutcheva, J. Phys. A 45, 125005 (2012). 
[35] C. Escudero, Phys. Rev. Lett. 101, 196102 (2008).

[36] E. Sherman and G. Pruessner, arXiv:1204.3017.

[37] U.C. Täuber, Critical Dynamics (Cambridge University Press, Cambridge, England, 2014).

[38] See Supplemental Material at http://link.aps.org/ supplemental/10.1103/PhysRevLett.121.020601 for further details.
[39] E. Frey and U. C. Täuber, Phys. Rev. E 50, 1024 (1994).

[40] R. Mannella, in Stochastic Processes in Physics, Chemistry, and Biology (Springer, New York, 2000), pp. 353-364.

[41] C. Escudero, F. Gazzola, and I. Peral, J. Math. Pures Appl. 103, 924 (2015).

[42] B. Chakrabarti and C. Dasgupta, Phys. Rev. E 69, 011601 (2004). 